\title{
Comparison of Radiological Parameters between Normal and Patellar Dislocation Groups in Korean Population: A Rotational Profile CT-Based Study
}

\author{
Jatin Prakash, $\mathrm{MD}^{1}$, Jong-Keun Seon, $\mathrm{MD}^{2}$, Seong-Hwan Woo, $\mathrm{MD}^{2}$, Cheng Jin, $\mathrm{MD}^{3}$, and Eun-Kyoo Song, $\mathrm{MD}^{2}$ \\ ${ }^{1}$ Department of Orthopedic Surgery, Lady Hardinge Medical College University of Delhi, New Delhi, India; ${ }^{2}$ Department of Orthopaedic Surgery, Chonnam National \\ University Bitgoeul Hospital, Gwangju, Korea; ${ }^{3}$ Department of Orthopedic Surgery, Chinese People’s Armed Police Force, Zhejiang Corps Hospital, Jiaxing, China
}

\begin{abstract}
Purpose: Patellofemoral instability is a common cause of anterior knee pain in adolescents and young adults. Most normal and pathological values for diagnosing patellofemoral instability are based on Western literature. We conducted this radiological study to determine normal values for different patellofemoral parameters in a Korean population and to evaluate their usefulness in diagnosis.

Materials and Methods: We retrospectively reviewed the rotational profile computerized tomography (CT) scans of the patellar dislocation and control groups. Trochlear, patellar, rotational profile, and trochleo-patellar alignment parameters were compared between the groups. Receiver operating characteristic curves were drawn for significant parameters, and sensitivity and specificity were calculated for the cut-off values.

Results: There were 48 patients in the patellar dislocation group and 87 patients in the control group. In the control group and patellar dislocation group, the mean sulcus angle was $132.5^{\circ}$ and $143.3^{\circ}$, respectively, trochlear depth was $6.04 \mathrm{~mm}$ and $3.6 \mathrm{~mm}$, bisect offset was $56.4 \%$ and $99.9 \%$, lateral patellar tilting was $9.8^{\circ}$ and $19.2^{\circ}$, patellar facet asymmetry was $63.5 \%$ and $45.16 \%$, and the tibial tuberosity-trochlear groove (TT-TG) distance was $10.91 \mathrm{~mm}$ and $27.16 \mathrm{~mm}$, respectively.

Conclusions: The trochlear depth, bisect offset, patella tilting, and TT-TG distance were parameters that significantly contributed to patellar instability. Rotational profile CT can be considered a good diagnostic tool to assess all these parameters that help to identify anatomical aberration resulting in patellofemoral instability, thereby helping in formulating the most effective treatment plan.
\end{abstract}

Keywords: Patella, Dislocation, Computerized tomography, Radiologic, Parameter

\section{Introduction}

Patellofemoral instability encompasses a wide spectrum of symptoms ranging from patellofemoral pain to overt patellar dislocation $^{1)}$. It is a common cause of anterior knee pain in adolescents and young adults, and patellar malalignment over trochlear

Received January 27, 2016; Revised March 31, 2016;

Accepted April 21, 2016

Correspondence to: Eun-Kyoo Song, MD

Department of Orthopaedic Surgery, Chonnam National University Bitgoeul Hospital, 80 Deongnam-gil, Nam-gu, Gwangju 61748, Korea Tel: +82-62-670-9475, Fax: +82-62-670-9476

E-mail: eksong@jnu.ac.kr

This is an Open Access article distributed under the terms of the Creative Commons Attribution Non-Commercial License (http://creativecommons.org/licenses/by-nc/4.0/) which permits unrestricted non-commercial use, distribution, and reproduction in any medium, provided the original work is properly cited. groove forms a precursor for early patellofemoral arthritis. The exact etiology is not well understood and it is assumed to be multifactorial resulting from a wide variety of anatomical aberrations that may be intra-articular (involving the trochlea and patella) or extra-articular based on anatomical orientation of the femur and tibia (excessive valgus alignment or rotation of bones in relation to each other) ${ }^{2-7)}$.

Dejour et al. ${ }^{8)}$ did one of the pioneer works on anatomic and radiological parameters of patellofemoral instability and they concluded 4 factors to be relevant to the pathology of patellar instability: trochlear dysplasia, quadriceps dysplasia, patella alta, and the tibial tuberosity-trochlear groove (TT-TG) distance. Since their work, there have been improvements in technology of image acquisition; particularly, image overlapping and rotational profile scans, which has led to the identification of a wide range of other parameters accounting for patellofemoral instability. A number of studies have tried to define these parameters through 
computerized tomography (CT) or magnetic resonance imaging $^{9-11)}$. Most of these studies have been conducted on American and European populations, and even among these studies, there are variations in the normal and cut-off values. For example, Alemparte et al. ${ }^{12)}$ studied healthy volunteers and found that normal values for TT-TG were $13.6 \pm 8.8 \mathrm{~mm}$, indicating a large variation. Dejour et al. ${ }^{8)}$ found that the TT-TG in their control group was $12.7 \pm 3.4 \mathrm{~mm}$ with a value of $>20 \mathrm{~mm}$ being considered pathological. Similarly, the lateral patella tilt angle documented in the study by Laurin et al. ${ }^{13)}$ was $8^{\circ}-13^{\circ}$, whereas Alemparte et al. ${ }^{12)}$ described $8.1^{\circ} \pm 14.5^{\circ}$ as normal values, showing a very wide variation. In addition, there is a paucity of such literature for Asian populations in particular. To the best of our knowledge, none of the studies in literature has yet determined the normal and cutoff values for patellofemoral and rotational profile parameters for Korean population.

We conducted this radiological study with the aim of 1) determining the normal values of different patellofemoral parameters in normal subjects, 2) comparing these values to those presented in literature, 3) investigating parameters that differ significantly in patellar dislocated patients, 4) identifying cut-off values of such parameters, and 5) evaluating their usefulness in the diagnosis of patellofemoral instability, which can further help to formulate an effective treatment plan. We did this by comparing the values on rotational profile CT scans between normal population and patients with patellar dislocation. Our hypothesis was that trochlear, patellar, and rotational profile parameters would differ considerably between the control group and the patellar dislocation group. Also, we hypothesized that the normal values of Korean population would be different from those presented in literature mostly based on Western population data.

\section{Materials and Methods}

In this retrospective study, we reviewed the images of patients who underwent a rotational profile CT scan at our institute for patellofemoral dislocation from January 2011 to December 2013. The review yielded 36 patients with unilateral pathology and 6 patients with bilateral patellofemoral dislocation. The normal side in unilateral cases was included in the analysis of the control group. We also reviewed the records of all patients for problems other than the knee joint. The values obtained from the normal side $(n=51)$ were used for the analysis of the control group.

Rotational profile CT scans were performed in all patients with similar equipment under similar conditions by a single radiologist not involved in this study. The position for CT scan was simi- lar in all cases and monitored strictly by an orthopedic resident and a radiographic technician. Patients lay in supine position with the knees in full extension. Using a hand-held goniometer, $15^{\circ}$ of external rotation was elicited. The angle made by the 2 nd toe to vertical was maintained at $15^{\circ}$ and the knee was strapped at mid-thigh and mid-leg using Velcro (Circaid Juxtafit; Medi $\mathrm{GmbH}$, Bayreuth, Germany) straps. After acquisition of images, we investigated different aspects on these images that can affect the patellofemoral articulation: lower limb alignment, trochlear parameters, patellar parameters, patellofemoral alignment, and rotational profile.

An independent senior surgeon, well versed in the method of measurement, recorded all parameters on 3 different occasions at least 2 weeks apart. The mean of these three values was taken as the final value. All values were rounded up to the second decimal point.

\section{Limb Alignment}

Excessive valgus alignment has often been regarded as a cause of patellofemoral malalignment. So, we analyzed the mechanical axis deviation and tibiofemoral angle. A line was drawn from the center of the femoral head to the center of the talar dome and its deviation from the center of notch was defined as mechanical axis deviation. The angle formed between the anatomical axis of the tibia and femur was measured as the tibiofemoral angle. These measurements were made on the scout film.

\section{Trochlear Parameters}

The trochlear study was conducted on the axial image that best described its anatomy. The best section was considered when the intercondylar notch took an appearance of a rounded roman arch. We studied 7 parameters in trochlear morphology (Fig. 1): 1) posterior condylar angle, 2) trochlear angle, 3) lateral trochlear inclination, 4) medial trochlear inclination, 5) sulcus angle, 6) trochlear depth, and 7) trochlear facet asymmetry defined as the ratio of the medial trochlear facet width to the lateral trochlear facet width.

\section{Patellar Parameters}

The morphology of the patella was analyzed on the axial cut with maximal patellar width. The width, height, and their ratio were recorded. Also, asymmetries of both patellar facets were assessed from the same film.

\section{Patellofemoral Alignment}

Alignment of the patella over the trochlea is an important as- 

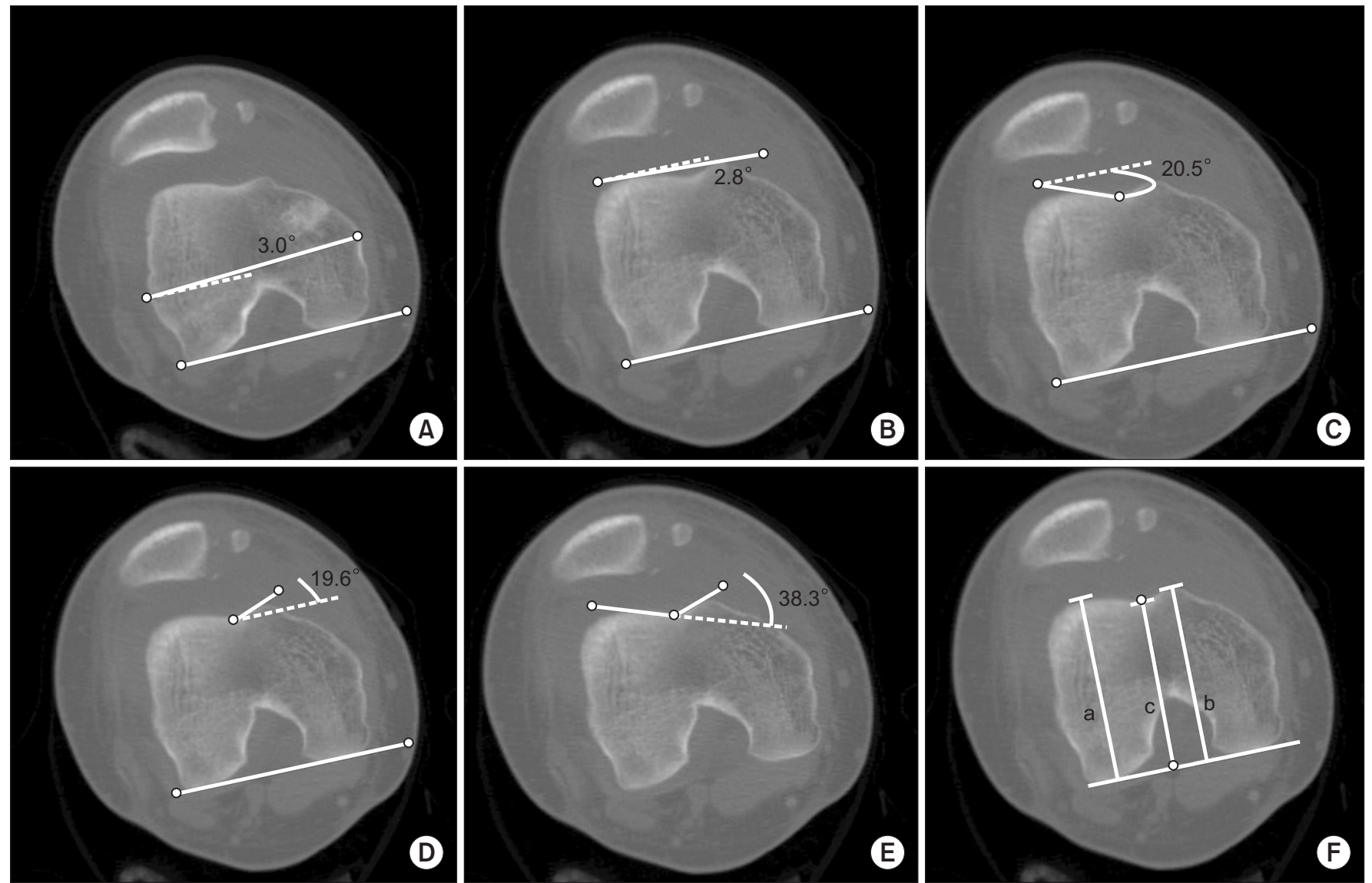

Fig. 1. (A) Posterior condylar angle: the angle between the posterior condylar line and the transepicondylar line. (B) Trochlear angle: the angle between the posterior condylar line and a tangential line passing through the anterior aspects of the medial and lateral trochlea. (C) Lateral trochlear inclination: the angle between the posterior condylar line and the lateral trochlear facet. (D) Medial trochlear inclination: the angle the posterior condylar line and the medial trochlear facet. (E) Sulcus angle: the angle between the medial and lateral trochlear facets. (F) Trochlear depth: the inset depth of the trochlear groove relative to the mean of the medial and lateral femoral condyle outsets calculated as $[(a-b)+(b-c)] / 2$. a: lateral facet height, b: medial facet height, c: perpendicular line from the sulcus to the posterior condylar line.
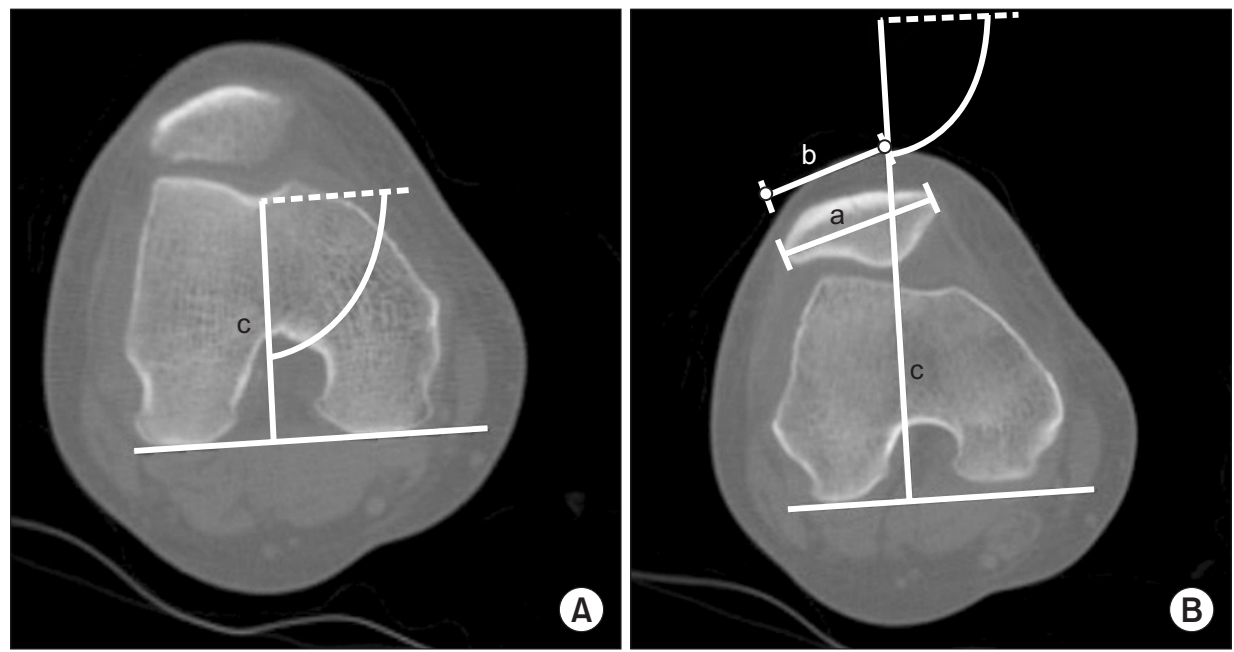

Fig. 2. Bisect offset. (A) Patellar and trochlear computed tomography images were different. The trochlear bisect line was drawn on the slice with the best trochlear profile. (B) The trochlear bisect line was extended on the patellar image. Patellar lines were drawn on the slice with maximum patellar width. c: trochlear bisect line drawn on the trochlear axial image, a: patellar width, b: the width of the patella lateral to the trochlear bisect line. Bisect offset is $\mathrm{b} /$ $\mathrm{a} \times 100$. 
pect that affects patellar tracking and may have a causative role in patellofemoral dysfunction. It was measured on the same slice that morphologic features of the trochlea were measured. On this slice, a perpendicular line was drawn through the center of the trochlea intersecting with the posterior condylar line. Then, on the slice where the patella width was maximal, a line connecting the medial and lateral margins of the patella was drawn. If the posterior condylar line was drawn on a different slice than the slice with maximal patellar width, the posterior condylar line was copied to the slice where the patella width was maximal. Different angles were noted to best define the patella-trochlear articulation: 1) bisect offset: it was defined as the percentage of the patella lateral to the line through the center of the trochlea (Fig. 2), 2) lateral patella tilt angle: it was defined as the angle between the posterior condylar line and the line defining the maximal width of the patell, 3) Laurin angle: it is the angle between the lines drawn along the lateral patellar facet and the anterior margins of femoral trochlea. 4) congruence angle: it is comprised between a line bisecting the sulcus angle and a second line joining the apex of trochlear groove with the apex of the patella.

\section{Rotational Profile Parameters}

Orientation of the femur with respect to the tibia may affect the

Table 1. Demographic Details of Two Groups

\begin{tabular}{lccc}
\hline \multicolumn{1}{c}{ Parameter } & $\begin{array}{c}\text { Patellar } \\
\text { dislocation } \\
\text { group }\end{array}$ & $\begin{array}{c}\text { Control } \\
\text { group }\end{array}$ & p-value \\
\hline Age (yr) & 23.43 & 25.60 & 0.76 \\
Sex (M:F) & $11: 31$ & $25: 62$ & $0.31^{\text {a) }}$ \\
Laterality (left:right) & $26: 22$ & $45: 42$ & $0.54^{\text {a) }}$ \\
Mechanical axis $\left({ }^{\circ}\right)$, mean (SD) & $6.84(2.1)$ & $5.43(1.9)$ & 0.56 \\
\hline
\end{tabular}

SD: standard deviation.

${ }^{a)}$ Chi-square test. patellofemoral articulation as well. An increase in valgus alignment may result in abnormal lateral pull of quadriceps; similarly, excessive rotations may disturb dynamic stabilizers in the knee joint. With this hypothesis, we measured following rotational parameters in both groups: 1) femoral anteversion: it was measured as the angle between the posterior condylar line and a line joining the center of the femoral head and the femoral neck, 2) tibial torsion: the angle formed by a tangent line to the posterior aspect of plateau on section in proximal tibial epiphysis just below articular surface and a tangent line through bimalleolar axis on the section near the ankle joint at the base of malleoli, 3) TT-TG distance: the distance was measured by superimposing the axial images that best represents the trochlea and the tibial tubercle and the distance was measured between the tibial tuberosity and the deepest point of the trochlear groove, 4) malleolar condylar angle: it is the angle between the posterior condylar line and a tangent line through the bimalleolar axis on the section near the ankle joint at the base of malleoli, 5) knee rotation angle: it is the angle between the posterior condylar line and a tangent line to posterior aspect of the plateau in the proximal tibial epiphysis just below the articular surface.

\section{Statistical Analysis}

The values obtained in two groups were analyzed by independent Student $t$-test to detect any statistically significant differences. The Mann-Whitney $U$-test was applied to variables with an asymmetric distribution. Cut-off values of the variables that showed significant difference between the groups were calculated as mean \pm twice standard deviation, and its effectiveness as a diagnostic test and sensitivity and specificity were assessed using receiver operating characteristic (ROC) curves. The area under the ROC curve was measured to classify the accuracy of a diagnostic test. The interpretation of the area was as follows: $0.90-1$, excellent diagnostic test; $0.80-0.90$, good diagnostic test; $0.70-0.80$, fair diagnostic test; $0.60-0.70$, poor diagnostic test; and $0.50-0.60$,

Table 2. Trochlear Parameters and ROC Curve Values for Parameters Significantly Different between Groups

\begin{tabular}{lcccc}
\hline \multicolumn{1}{c}{ Parameter } & Patellar dislocation group & Control group & p-value & ROC \\
\hline Sulcus angle $\left(^{\circ}\right)$ & $143.32(33.7)$ & $132.5(8.2)$ & 0.16 & N/A \\
Lateral trochlear inclination $\left(^{\circ}\right)$ & $16.76(5.6)$ & $21.85(5.5)$ & 0.04 & 0.679 \\
Medial trochlear inclination $\left(^{\circ}\right)$ & $17.35(10.3)$ & $19.84(6.7)$ & 0.37 & N/A \\
Facet asymmetry & $45.16(14.6)$ & $63.50(14.9)$ & 0.01 & 0.712 \\
Trochlear depth & $3.60(0.8)$ & $6.04(0.6)$ & 0.00 & 0.852 \\
Posterior condylar angle $\left(^{\circ}\right)$ & $5.80(2.7)$ & $4.60(1.9)$ & 0.13 & N/A \\
\hline
\end{tabular}

Values are presented as mean (standard deviation).

ROC: receiver operating characteristic, N/A: not applicable. 
fail. To calculate the sample size to assess the adequacy of ROC, we did a power analysis with MedCalc ver. 16.2 (MedCalc Software, Ostend, Belgium). An alpha error set at 0.05 and beta error at 0.80 with estimated ROC curve of 0.700 gave a sample size of 38 in each group. Therefore, our sample size was appropriate.

A multiple logistic regression model was used to identify the most significant variables for occurrence of patellar dislocation. All analyses were performed using a two- tailed test.

\section{Results}

Rotational profile CT scans were compared between the two groups: dislocated group ( $\mathrm{n}=48,36$ unilateral and 6 bilateral) and control group ( $\mathrm{n}=87,36$ normal sides of the dislocated patients and 51 patients with problems other than the knee). Table 1 shows the demographic data of both groups. Agreement between all 3 values of each parameter recorded by the surgeon was found excellent $(\mathrm{p}=0.000)$.

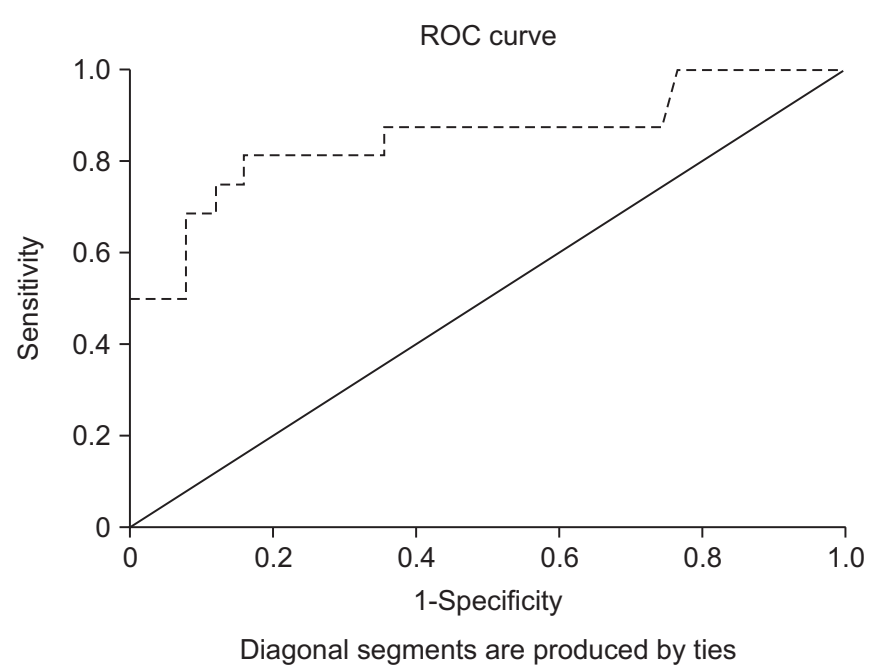

Fig. 3. Receiver operating characteristic (ROC) curve for trochlear depth with the area under the curve being 0.852 .

\section{Limb Alignment}

The average tibiofemoral angle was $6.84^{\circ} \pm 2.1^{\circ}$ valgus in the dislocation group and $5.43^{\circ} \pm 1.9^{\circ}$ valgus in the control group showing no statistically significant difference $(\mathrm{p}=0.56)$. No significant mechanical axis deviation was observed in both groups.

\section{Trochlear Parameters}

Lateral trochlear inclination, facet asymmetry and trochlear depth values were found to be significantly different in two groups. Other trochlear values were not found significantly different. Table 2 shows the mean values and standard deviations of trochlear parameters.

In order to determine the diagnostic value for evaluating patellar subluxation, ROC curves were drawn for the significantly different variables in two groups. The values are presented in Table 2 . Trochlear depth was the best test for diagnosis among trochlear parameters with 0.852 under the curve area, indicating it is a very good diagnostic test (Fig. 3). A cut-off value of $4.75 \mathrm{~mm}$ (mean standard deviation of normal population) was $81 \%$ sensitive and $84 \%$ specific.

\section{Patellar Parameters}

The means of patella width, height and facet asymmetry were significantly different; however, the ratio of width to height was not found to be statistically significantly different between the groups. Table 3 shows the mean and standard deviation of patellar parameters in both groups. Asymmetry of patellar facets was found to be a very good diagnostic test with 0.866 under the curve area in the ROC analysis. A cut-off of $55 \%$ (width of the medial facet compared to the lateral) yielded a specificity of $100 \%$ and sensitivity of $25 \%$.

\section{Patellofemoral Alignment}

Means of Laurine angle, patellar tilt angle, congruence angle, and bisect offset were all statistically significantly different. Table 4 shows the means of these parameters. Patellar tilt angle and bisect offset were of most diagnostic values among these parame-

Table 3. Patellar Parameters and ROC Curve Values for Parameters Significantly Different between Groups

\begin{tabular}{lcccc}
\hline \multicolumn{1}{c}{ Parameter } & Patellar dislocation group & Control group & p-value & ROC \\
\hline Patella facet asymmetry & $65.3(17.62)$ & $91.4(18.20)$ & 0.00 & 0.866 \\
Patella width $(\mathrm{mm})$ & $39.1(3.50)$ & $43.9(3.30)$ & 0.00 & 0.512 \\
Patella height $(\mathrm{mm})$ & $18.3(2.09)$ & $21.0(2.98)$ & 0.00 & 0.645 \\
Width to height ratio & $2.13(0.24)$ & $2.12(0.43)$ & 0.89 & N/A \\
\hline
\end{tabular}

Values are presented as mean (standard deviation).

ROC: receiver operating characteristic, N/A: not applicable. 
Table 4. Patellofemoral Alignment Parameters and ROC Curve Values for Parameters Significantly Different between Groups

\begin{tabular}{lcccc}
\hline \multicolumn{1}{c}{ Parameter } & Patellar dislocation group & Control group & p-value & ROC \\
\hline Patella tilt angle $\left(^{\circ}\right)$ & $29.11(8.1)$ & $5.77(3.8)$ & 0.000 & 0.974 \\
Laurine angle $\left(^{\circ}\right)$ & $19.2(2.4)$ & $9.8(2.1)$ & 0.020 & 0.843 \\
Congruence angle $\left(^{\circ}\right)$ & $23.85(1.3)$ & $3.2(1.1)$ & 0.000 & 0.713 \\
Bisect offset $(\%)$ & $99.9(10.2)$ & $56.4(14.3)$ & 0.000 & 0.985 \\
\hline
\end{tabular}

Values are presented as mean (standard deviation). ROC: receiver operating characteristic.

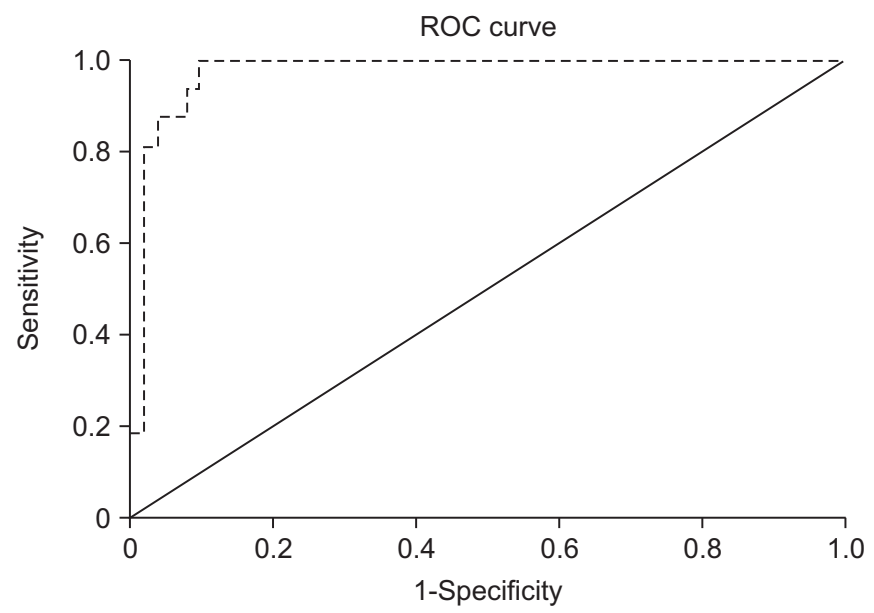

Fig. 4. Receiver operating characteristic (ROC) curve for bisect offset with the area under the curve being 0.985 .

ters. A cut-off value of over $13.5^{\circ}$ for patellar tilting showed a sensitivity of $100 \%$ and specificity of $91 \%$. The area under the ROC curve was 0.974 , indicating it is an excellent diagnostic test. Bisect offset also had an excellent diagnostic value with 0.985 as an area under the curve (Fig. 4). A value of $85 \%$ or above when chosen as a cut-off for patellar dislocation had a specificity of $100 \%$ and sensitivity of $92 \%$.

\section{Rotational Profile Parameters}

Mean values of tibial torsion and femorotibial index were not significantly different in two groups; however, TT-TG, femoral anteversion, knee rotation angle, and malleolar condylar angle were found to be remarkably different between the groups. Table 5 summarizes the results. The ROC curve showed TT-TG to be the best indicator of patellar dislocation with 0.966 under the curve area with a value of $20.5 \mathrm{~mm}$ as $94 \%$ sensitive and $97 \%$ specific for patellar dislocation (Fig. 5). Knee rotation angle also served as a very good diagnostic test with a value of 0.884 as the under the curve area. Malleolar condylar angle and femoral anteversion, though significantly different in both groups, had little diagnostic value in ROC curves.

Multiple regression analysis showed that trochlear depth, troch- lear facet asymmetry; bisect offset, patellar tilt angle, and TT-TG were the most important variables contributing to dislocation of the patella.

\section{Discussion}

Our study showed that among various parameters, trochlear depth, bisects ratio, lateral patellar tilting, patellar facet asymmetry, and TT-TG distance were the best markers for the diagnosis of patellar instability. Also, the logistic regression analysis showed that the TT-TG distance; bisect offset, and patellar tilting were the most important predictors of patellar dislocation having the highest risk ratio.

The etiology of patellofemoral instability is multifactorial and has encouraged many authors to carry out radiological studies to define the anatomical basis of the disease and formulate appropriate treatment plans ${ }^{2-8)}$. Due to the advancement in image acquisition technology, high quality images can be obtained without distortion. In addition, the image overlay technique has improved understanding of the etiology of this complex syndrome. A number of new parameters have been introduced and attempts have been made to analyze rotational profile of the lower limb as an etiological factor. Many reports have demonstrated the superiority of CT scan in analyzing the patellofemoral joint in knee extension, which is impossible with radiography ${ }^{9-11)}$.

Dejour et al. $^{8)}$ have regarded trochlear dysplasia as a pathognomonic feature of symptomatic pain syndrome. However, in our study, we found inconsistent results for parameters of trochlear morphology. Trochlear angle was not significantly different between the two groups and although sulcus angle was notably different, it had minimal role in diagnosing patellar instability. Other authors have also found trochlear dysplasia as an inconsistent feature $^{14-16)}$. Since patellofemoral instabilities are multi-factorial, we believe that the interrelationship of both patella and trochlea is more important than dysplasia of trochlea alone. Among all trochlear parameters we found, trochlear depth appeared to have the most consistent effect on patellofemoral dislocation. We found a value of less than $3.5 \mathrm{~mm}$ to be $100 \%$ specific for patellar 
Table 5. Rotational Profile Parameters and ROC Curve Values for Parameters Significantly Different between Groups

\begin{tabular}{|c|c|c|c|c|}
\hline Parameter & Patellar dislocation group & Control group & $\mathrm{p}$-value & ROC \\
\hline Tibial tuberosity-trochlear groove & $27.16(5.5)$ & $10.91(4.8)$ & 0.000 & 0.966 \\
\hline Femoral anteversion & $19.2(10.4)$ & $12.0(8.4)$ & 0.010 & 0.698 \\
\hline Knee rotation angle $\left(^{\circ}\right)$ & $11.5(4.5)$ & $4.8(2.8)$ & 0.000 & 0.844 \\
\hline Tibial torsion & $31.4(7.4)$ & $30.6(4.4)$ & 0.770 & N/A \\
\hline Malleolar condylar angle $\left(^{\circ}\right)$ & $37.9(5.3)$ & $27.9(4.5)$ & 0.000 & 0.755 \\
\hline
\end{tabular}

Values are presented as mean (standard deviation).

ROC: receiver operating characteristic, N/A: not applicable.

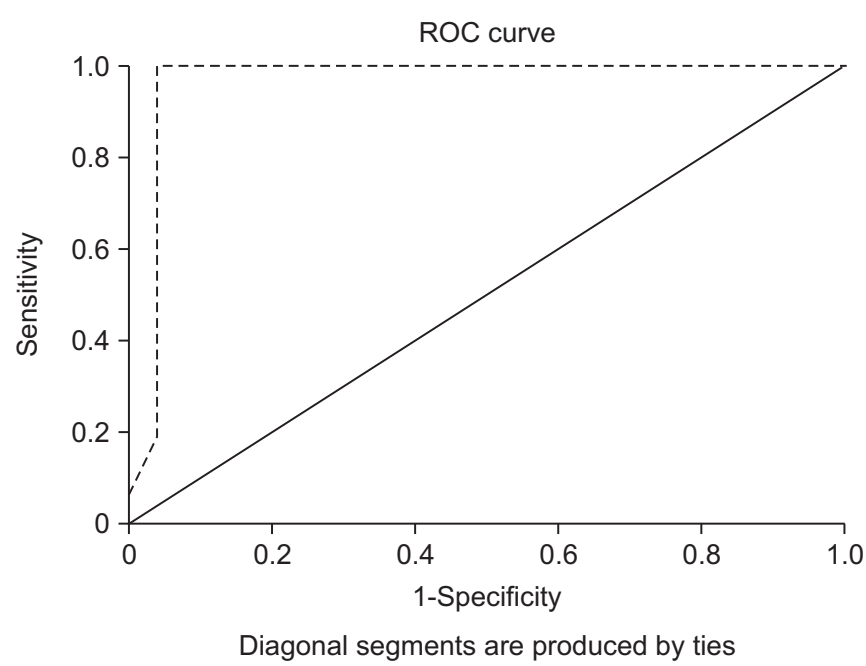

Fig. 5. Receiver operating characteristic (ROC) curve for tibial tuberosity-trochlear groove with the area under the curve being 0.966 .

dislocation. This was marginally different from the normal cutoff value of $3 \mathrm{~mm}$ in most studies ${ }^{12,15}$.

It was notable that the dislocated patella was significantly smaller in both height and width than the corresponding control group, but it did not present any diagnostic value. However, similar to Weiberg ${ }^{17)}$, we found that patella facet asymmetry was most consistently associated with patellar dislocation. The dislocated patellae tend to have a markedly small and convex medial facet compared to the lateral facet.

The parameters of patellofemoral alignment that primarily expressed the quadriceps dysplasia as lateralization of the patella was an objective flaw in extensor mechanism and these parameters were found to be the most consistent factors in a dislocated patella ${ }^{8)}$. There has been controversy regarding assessment of these parameters before and after quadriceps contraction. Few authors have suggested the values are different while others have found no difference in these values ${ }^{18-20)}$. We, however, agree with Martinez et al. ${ }^{20}$ who considered two imaging studies unnecessary, and preferred an assessment with a relaxed muscle, since it was easier to perform. We found bisect offset and patellar tilting angle most consistently associated with the dislocated patella. Apart from their diagnostic value, they were very important predictors of patellar dislocation and had a high relative risk ratio on logistic regression analysis. Their diagnostic value and high relative risk have been emphasized and established in various studies in the literature.

Increased lateralization of the tibial tuberosity can be evaluated by TT-TG. TT-TG is an absolute value independent of patient's height and weight ${ }^{8)}$. In this study, TT-TG was the most specific and sensitive method for detecting patellar instability and was accurate for predicting the risk of patellar dislocation in patients.

Rotational abnormalities may be a secondary cause of disorder in the extensor mechanism and have, therefore, been proposed as possible etiologies in patellofemoral instabilities. We found values of tibial torsion were remarkably similar between the groups; however, femoral anteversion, knee rotation angles and malleloar condylar angles were significantly different. Despite the difference in these groups, these values were not important in diagnosing dislocation except for knee rotation angle. This was similar to findings of Schueda et al. ${ }^{21)}$ who also found the means of rotational parameters to be significantly different. However, in contrast, they failed to show any diagnostic importance of these parameters. We found knee rotational angle consistently increased in patients with dislocated patellae.

Most studies on normal values are based on measurements of Western population. Therefore, the cut-off values have also been derived from the similar data. In the absence of database for Asian population, most surgeons have to rely on the data obtained from different ethnic groups both for the diagnosis and treatment. It is, however, clear that Asian knees differ from those of their Western counterpart ${ }^{22-24}$. Table 6 shows the comparison of values of various parameters between the established literature based on Western population and the current study, ${ }^{8,12,13,25-30)}$. The table further stresses the need to have a local database for diagnosis and treatment purposes as most variables are shown to differ 
Table 6. Comparison of Various Parameters Available in Literature and in the Present Study

\begin{tabular}{|c|c|c|c|c|}
\hline Parameter & Previous European/American study & Patellar dislocation group & Current study & Patellar dislocation \\
\hline Sulcus angle $\left({ }^{\circ}\right)$ & $123(5.1)$ & $>140$ & $132.5(8.2)$ & $143.32(33.7)$ \\
\hline Lateral trochlear inclination $\left(^{\circ}\right)$ & $22.1(9)$ & $<11$ & $21.85(5.5)$ & $16.76(5.6)$ \\
\hline Facet asymmetry $(\%)$ & 57 & 12 & 63.5 & $45.16(14.6)$ \\
\hline Trochlear depth (mm) & 5.2 & 0.6 & 6.04 & $3.6(0.81)$ \\
\hline Patella tilt angle $\left(^{\circ}\right)$ & $11.1(10.6)$ & $22.2(9.1)$ & $5.77(3.8)$ & $29.11(8.1)$ \\
\hline Laurine angle $\left(^{\circ}\right)$ & $8.1(14.5)$ & $3(11.1)$ & $9.8(2.1)$ & $19.2(2.4)$ \\
\hline Congruence angle $\left(^{\circ}\right)$ & $-6(11)$ & 23 (>16 abnormal) & $-3.2(1.1)$ & $23.85(1.3)$ \\
\hline Bisect offset (\%) & $58.7(9.4)$ & N/A & $56.4(14.3)$ & $99.9(10.2)$ \\
\hline $\begin{array}{l}\text { Tibial tuberosity-trochlear groove } \\
(\mathrm{mm})\end{array}$ & $12.4(2.4)$ & $\begin{array}{c}17.8 \\
(4.5 ;>20 \mathrm{~mm} \text { abnormal })\end{array}$ & $10.91(4.8)$ & $27.16(5.5)$ \\
\hline Femoral anteversion & $18.9(8.7)$ & $23.2(8.5)$ & $12.0(8.4)$ & $19.2(10.4)$ \\
\hline Knee rotation angle $\left(^{\circ}\right)$ & $4.7(3.2)$ & $6.8(4.5)$ & $4.8(2.8)$ & $11.5(4.5)$ \\
\hline Tibial torsion $\left(^{\circ}\right)$ & $32.5(5.8)$ & $32.7(7.7)$ & $30.6(4.4)$ & $31.4(7.4)$ \\
\hline Malleolar condylar angle $\left(^{\circ}\right)$ & $34.8(6.7)$ & $39.6(9.2)$ & $27.9(4.5)$ & $37.9(5.3)$ \\
\hline
\end{tabular}

Values are presented as mean (standard deviation).

N/A: not available.

between Asian and Western knees. For example, knee rotation angles have not been found abnormal in studies published so far based on the Western knees. However, we found knee rotation was consistently high in Korean knees. Tensho et al. ${ }^{30)}$ observed a similar finding of knee rotation in Japanese knees. We believe that data from multiple centers on the normal and cut-off values may help to build a database that would serve as a guidance for better diagnosis and treatment particularly for Asian patients.

Understanding of all above parameters is important to formulate a treatment plan that is most suited for patients. The existence of an excessive patellar tilt may indicate abnormalities in quadriceps mechanism and flexion of the knee may not be restored despite centering of the patella over the trochlea as quadriceps remains tight. Some form of quadricepsplasty may be necessary in these cases. Similarly, a high TT-TG distance may be an indication of a distal-based procedure requiring medicalization of the tibial tuberosity. Increased knee rotation or femoral anteversion may dictate a rotational osteotomy at respective anatomical site. A highly distorted trochlear angle or dysplastic trochlea would require a trochleoplasty. Rotational profile can exactly identify anatomical aberration and an extensive study of these scans, therefore, is necessary to individualize the procedure for each case.

Our study has a few limitations. First, the sample size was small and thus it was difficult to extrapolate the results to general population. Studies from other Asian centers are necessary to build up a regional anthropometric database and the current study was a preliminary step to this end. Second, we did not have symptomatic nondislocated group in this study; comparison of a completely dislocated group to the control group may show remarkable differences in values of few parameters. Inclusion of a symptomatic non-dislocated group would be helpful in better evaluation of diagnostic usefulness of these parameters. Third, this study has a retrospective design that is less reliable than a prospective randomized study.

\section{Conclusions}

Rotational profile CT should be part of a routine examination to determine the diagnosis and treatment plans in patellar dislocation patients. We found trochlear depth; bisect offset, patella tilting, and TT-TG distance to be the most significant parameters contributing to patellar instability. Rotational profile CT can be useful for the identification of anatomical aberration associated with patella-femoral instability and thereby helps to formulate the most effective treatment plan. This study also demonstrated the normal values of various patellofemoral parameters in the Korean population are different from those in Western populations.

\section{Conflict of Interest}

No potential conflict of interest relevant to this article was reported. 


\section{References}

1. Galland O, Walch G, Dejour H, Carret JP. An anatomical and radiological study of the femoropatellar articulation. Surg Radiol Anat. 1990;12:119-25.

2. Aglietti P, Buzzi R,Insall JN. Disorders of the patellofemoral joint. In: Insall JN, Scott WN, eds. Surgery of the Knee. 3rd ed. New York: Churchill Livingstone; 2001. p246-51.

3. Aulisa AG, Falciglia F, Giordano M, Savignoni P, Guzzanti V. Galeazzi's modified technique for recurrent patella dislocation in skeletally immature patients. J Orthop Sci. 2012;17:148-55.

4. Biedert RM, Bachmann M. Anterior-posterior trochlear measurements of normal and dysplastic trochlea by axial magnetic resonance imaging. Knee Surg Sports Traumatol Arthrosc. 2009;17:1225-30.

5. Blumensaat C, Ergenbn D. Patellofemoral disorders: physical and radiographic evaluation. Chir U Orthop. 1938;31:149223.

6. Colvin AC, West RV. Patellar instability. J Bone Joint Surg Am. 2008;90:2751-62.

7. Conlan T, Garth WP Jr, Lemons JE. Evaluation of the medial soft-tissue restraints of the extensor mechanism of the knee. J Bone Joint Surg Am. 1993;75:682-93.

8. Dejour H, Walch G, Nove-Josserand L, Guier C. Factors of patellar instability: an anatomic radiographic study. Knee Surg Sports Traumatol Arthrosc. 1994;2:19-26.

9. Inoue $\mathrm{M}$, Shino K, Hirose $\mathrm{H}$, Horibe $\mathrm{S}$, Ono K. Subluxation of the patella. Computed tomography analysis of patellofemoral congruence. J Bone Joint Surg Am. 1988;70:1331-7.

10. Insall J, Salvati E. Patella position in the normal knee joint. Radiology. 1971;101:101-4.

11. Martinez S, Korobkin M, Fondren FB, Hedlund LW, Goldner JL. Diagnosis of patellofemoral malalignment by computed tomography. J Comput Assist Tomogr. 1983;7:1050-3.

12. Alemparte J, Ekdahl M, Burnier L, Hernández R, Cardemil A, Cielo R, Danilla S. Patellofemoral evaluation with radiographs and computed tomography scans in 60 knees of asymptomatic subjects. Arthroscopy. 2007;23:170-7.

13. Laurin CA, Dussault R, Levesque HP. The tangential x-ray investigation of the patellofemoral joint: $\mathrm{x}$-ray technique, diagnostic criteria and their interpretation. Clin Orthop Relat Res. 1979;(144):16-26.

14. Harbaugh CM, Wilson NA, Sheehan FT. Correlating femoral shape with patellar kinematics in patients with patellofemoral pain. J Orthop Res. 2010;28:865-72.
15. Stefanik JJ, Zumwalt AC, Segal NA, Lynch JA, Powers CM. Association between measures of patella height, morphologic features of the trochlea, and patellofemoral joint alignment: the MOST study. Clin Orthop Relat Res. 2013;471:2641-8.

16. Haim A, Yaniv M, Dekel S, Amir H. Patellofemoral pain syndrome: validity of clinical and radiological features. Clin Orthop Relat Res. 2006;451:223-8.

17. Wiberg G. Roentgenographs and anatomic studies on the femoropatellar joint: with special reference to chondromalacia patellae. Acta Orthop Scand. 1941;12:319-410.

18. Grelsamer RP, Klein JR. The biomechanics of the patellofemoral joint. J Orthop Sports Phys Ther. 1998;28:286-98.

19. Guzzanti V, Gigante A, Di Lazzaro A, Fabbriciani C. Patellofemoral malalignment in adolescents: computerized tomographic assessment with or without quadriceps contraction. Am J Sports Med. 1994;22:55-60.

20. Martinez S, Korobkin M, Fondren FB, Hedlund LW, Goldner JL. Diagnosis of patellofemoral malalignment by computed tomography. J Comput Assist Tomogr. 1983;7:1050-3.

21. Schueda MA, Astur DC, Bier RS, Bier DS, Astur N, Cohen M. Use of computed tomography to determine the risk of patellar dislocation in 921 patients with patellar instability. Open Access J Sports Med. 2015;6:55-62.

22. Maas A, Kim TK, Miehlke RK, Hagen T, Grupp TM. Differences in anatomy and kinematics in Asian and Caucasian TKA patients: influence on implant positioning and subsequent loading conditions in mobile bearing knees. Biomed Res Int. 2014;2014:612838.

23. Yue B, Varadarajan KM, Ai S, Tang T, Rubash HE, Li G. Differences of knee anthropometry between Chinese and white men and women. J Arthroplasty. 2011;26:124-30.

24. Kim TK. Special considerations for TKA in Asian patients: editorial comment. Clin Orthop Relat Res. 2013;471:143940.

25. Diederichs G, Issever AS, Scheffler S. MR imaging of patellar instability: injury patterns and assessment of risk factors. Radiographics. 2010;30:961-81.

26. Laurin CA, Levesque HP, Dussault R, Labelle H, Peides JP. The abnormal lateral patellofemoral angle: a diagnostic roentgenographic sign of recurrent patellar subluxation. J Bone Joint Surg Am. 1978;60:55-60.

27. Merchant AC, Mercer RL, Jacobsen RH, Cool CR. Roentgenographic analysis of patellofemoral congruence. J Bone Joint Surg Am. 1974;56:1391-6.

28. Fulkerson JP, Buuck DA. Disorders of the patellofemo- 
ral joint. 4th ed. Philadelphia, PA: Lippincott Williams \& Wilkins; 2004. p211-39.

29. Endo Y, Stein BE, Potter HG. Radiologic assessment of patellofemoral pain in the athlete. Sports Health. 2011;3:195-210.

30. Tensho K, Akaoka Y, Shimodaira H, Takanashi S, Ikegami
S, Kato H, Saito N. What components comprise the measurement of the tibial tuberosity-trochlear groove distance in a patellar dislocation population? J Bone Joint Surg Am. 2015;97:1441-8. 